\title{
Empricial Analysis on Influencing Factors and Effects of Political Education Micro-Blogs
}

\author{
Tang Yeren*
}

Hunan Mechanical and Electrical Polytechnic, Hunan Changsha 410151 China

Hunan Normal University Academy of Educational, Hunan Changsha 410151 China

\begin{abstract}
Information broadcasting of micro-blog network can help users to acknowledge its broadcasting characteristics and rules, to provide theoretical basis and practical instruction for scientific monitoring and dissemination of public opinions. This paper adopts micro-blogs of SINA website as the research object to analyze the topology feature and information broadcasting rules of user. It makes a statistics on network topology of confusion network composed by social users, and verifies the influence on information broadcasting caused by network structure. Then it further studies the transmission characteristics and comment characteristics micro-blog messages, providing the influencing factors of broadcasting network with given cases. During the experimental process we analyze the broadcasting effects under different conditions, showing the characteristic of our model is in accordance with real situation.
\end{abstract}

Keywords: Micro-blog, Influencing factor, Transmission, Broadcasting model, Social network.

\section{INTRODUCTION}

As a rising network communication tool, micro-blog has characteristics like fast communication, convenient operation, massive information, low cost, etc. It has become self-catharsis and self-expression space in public in the form of convenient publication, autonomous concern and acquisition. Furthermore, its chain communication model focuses on information affects people's communications. It triggers forming as well as development of social public opinion and new tendency of communication to some extent. People have diversification tendency on the thought as well as values and provide new perspective, new concept and new mode for political work in the new age. It is a novel topic on how to actively explore and treat new challenges brought by micro-blog and master, as well as implement various new technology and new measures to apply creative political work style by micro-blog to finally become new subject [1]. Users under micro-blog platform are not only information receivers but also information transmitters. Users broadcast the information mainly by publishing original information and transmitting others' information. Micro-blog information broadcasting relies on users' transmitting behavior. By means of studying users' transmitting behavior, users' relationship, internal relationship between micro-blog contents and transmitting rule of information on micro-blog, relative research tries to find kernel influencing factors during information transmission to predict transmitting number of information.
Recently, domestic and foreign scholars make further study on information transmission models in social network.

There are two aspects: From macro aspect, scholars apply complex networked transmission dynamics model to analyze information transmission law in social network; From micro aspect, scholars analyze micro-blog users' transmitting behavior and study mechanism as well as law of information transmission in micro-blog. Yuan put forward a definition model and comprehensively reveal the reasons that consumers use micro-blog [2]. Their researches believe that there are four factors affecting users' micro-blog usage which are the perceived requirement, user's creativity, creative characteristics perception and popularity degree perception of micro-blog. The research emphasizes on users' psychology, website characteristics and other media influence. Most researches adopt qualitative research while quantitative researches are fewer. Thus, related further micro-blog research can adopt quantitative research method. Domestic scholars also attempt to summarize micro-blog transmission features. For instance, Liu summarized microblog transmission features as content minimization, medium movement and communication fragmentation [3]. Chen stresses that micro-blog has obvious 4A features: anyone, Anywhere, Anything and Anytime [4]. Yuan starts from public policy speech, analyzes transmission features and transmission modes of micro-blog through studying relative cases of SINA micro-blog and evaluates effects of microblog to construct public discourse platform [5].

Based on above literatures, it can be inferred that the establishment of micro-blog information transmission model is very complicated. Since different models need consider different nodes and file attribute features, node influence can 
select appropriate attribute features to calculate its values through improving current model algorithm. This paper adopts social network analysis to analyze micro-blog users' network structure and transmitting network structure of information. Meanwhile, it also uses statistics method to analyze transmission features as well as laws of information in micro-blog and behavior difference of users' publishing, reading and transmitting information. By characteristics analysis of micro-blog users' practical behavior, this paper integrates information transmission features to construct transmission model of micro-blog information. Through simulating experiment to analyze users' effect in information transmission, this paper verifies the information transmission characteristics and predicts the scope of information transmission. The rest of the paper is organized as follows: In section 2, we introduce the network topology of microblog network and the charactistics of information broadcastikng. In section 3, this paper analyzes the influencing factors on information broadcasting and proposed an improved broadcasting based on social network. In section 4 , we test the feasibility and practicability by experimental results. Finally, a conclusion is provided in section 5 .

\section{MICRO-BLOG NETWORK TOPOLOGY AND INFORMATION TRANSMISSION CHARACTERIS- TICS ANALYSIS}

Illustrated by SINA micro-blog, its opening platform promotes that developers can compile various application programs by providing programming access of application project for third party development staff, to realize seamless connection of micro-blog in order to reach various purposes. We recognize whether one registered users belong to educational type though two fields verified and verified_edu of users/show in SINA micro-blog API.

By means of concerned behavior, micro-blog user connects itself with other users to form a user's relationship network in complex structure. This relationship network can be expressed by directed graph $G(V, E)$. Node set $V$ denotes all users in micro-blog network, edge set $E$ is connection edge between nodes in micro-blog network, which denotes friend relationship and following relationship between two users. We will construct the social network topology structure figure based on acquired 50 samples and take it as one sub-network in social network of SINA micro-blog.

In above (Fig. 1), some of these users have relatively more in-degree nodes. The figure reflects there are many line connections of users' representing nodes. Line connections are very dense so users' relationship is very closely. However, some users have less nodes in-degree. Based on above figure, connection lines of these nodes are less and sparse.

\section{Characteristics Analysis}

By analyzing out-in degree in micro-blog network, it can be known whether it follows power-law distribution. Powerlaw distribution is also called scale-free distribution and the network with power-law degree distribution is also called scale-free network. However, scale-free characteristic is an important factor in complicated network. One way to describe the degree data is drawing accumulation degree distribution function:

$P_{k}=\sum_{k^{\prime}=k}^{\infty} P\left(k^{\prime}\right)$

It depicts the degree which is not smaller than $k$ node probability distribution. Because power-law distribution should basically correspond to one line in dual-logarithm coordinates system, it is very easy to use logarithmic coordinates and semilogarithmic coordinate to recognize power-law distribution.Pajek is used to calculate out-in degree and average degree number of data and its accumulating degree curve is also drawn. Fig. (2) shows accumulating degree distribution curve of networked indegree and accumulating degree distribution curve of

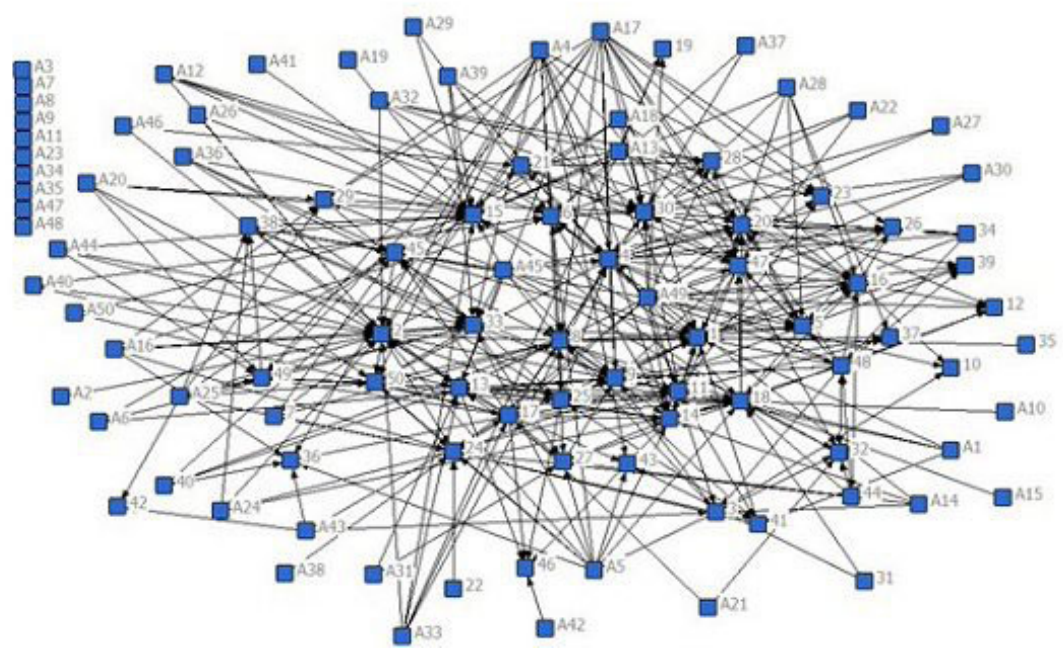

Fig. (1). Social network structure of SINA micro-blogs. 
networked out-degree. The result shows that in-degree distribution of micro-blog network basically matches powerlaw distribution.
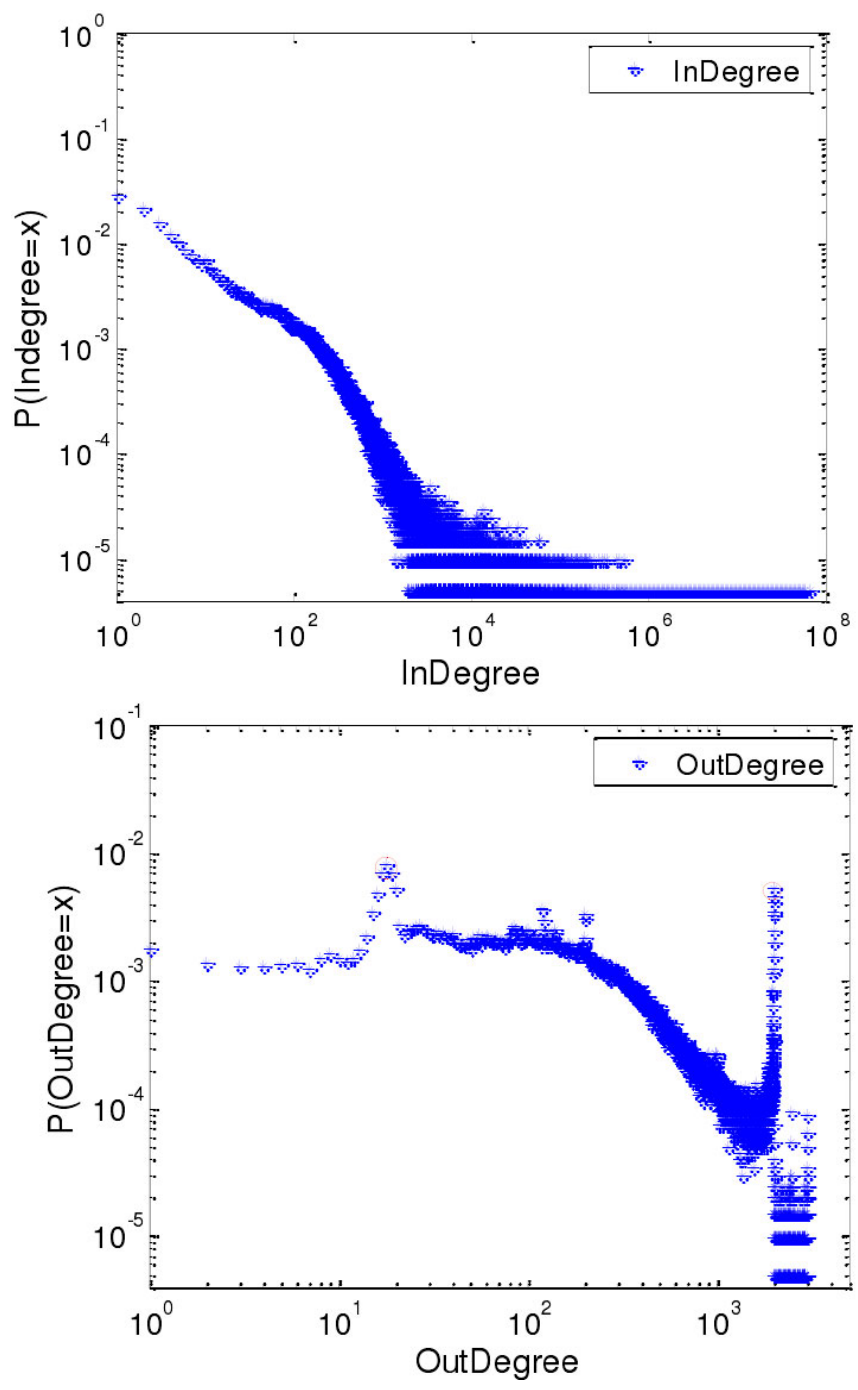

Fig. (2). Input and output distribution of micro-blog users.

In complicated network, defining the shortest path between nodes refers to one path with the least edge numbers from one node to another node to express accessibility between two nodes. That is, it is the shortest path to communicate information between two nodes. Average path length in network refers to the average value of the shortest path length between all node pairs in network while network diameter refers to the maximum value of the shortest path length between random two nodes in the network. Clustering coefficient in network is average value of all nodes clustering coefficient and it indicates close degree of connection between nodes in network. The larger this value, the closer the relationship between nodes, the higher the networked clustering degree. This paper analyzes that average path length in network is 4.33 , diameter is 12 and cluster coefficient is 0.229 . Table 1 shows measurement results of other social network topology structure in domestic and foreign researches.
By comparison and analysis, it can be found that average path length and diameter of SINA micro-blog are all very small and clustering coefficient is very large. It has smallworld characteristics and it indicates that users in SINA micro-blog are closely connected so the path of information communication is very short to be beneficial for fast communication of information [6-9].

\section{IMPROVED MICRO-BLOG INFORMATION TRANS- MISSION MODEL}

This paper puts forward three users' behavior characteristics: users' influence, reading habit and transmitting preference degree. Meanwhile, by statistical analysis, this paper uses users' historical data to describe users' behavior characteristics. Users' influence is calculated through the transmitted number of users' historical blog. The reading preference is described by time habit of users' historical blog and users' transmitting preference is determined according to users' historical transmitting evaluation.

\section{Users' Influence}

Set addition of original micro-blog number, micro-blog transmitting number and evaluation number as active degree of node, which is expressed by active. Set addition of average re-transmitted number of original micro-blog and the evaluated number as spreading capacity of nodes, which is expressed as spread. Active fans each day is taken as node coverage degree, which is expressed as coverage. Transmitting influence influence is obtained by formula 2 and $\alpha, b, c$, are coefficients.

influence $=\alpha^{*}$ active $+b^{*}$ spread $+c^{*}$ coverage

The influence of key nodes is different when they are taken as secondary transmitting node and original node. The average micro-blog transmitting number of acquired key node within certain time AR is taken as measurement value to affect factors. Due to sudden event occurrence, the heat level of this event is different from common information communication. The proposed background of this model does not consider micro-blog transmitting times about sudden events of key nodes. AR refers to node's average value of micro-blog transmitting times which is transmitted within certain time.

\section{Reading Preference}

After user issues the information on micro-blog, his blog will all be pushed to his fans' index page. In general, as long as fans $\log$ in personal page, they will read the newly occurred events at first page. Thus, it can be induced that the smaller the time interval between users' log-in time and his friends' blogs, the larger the possibility users read friends' blog. However, it is very difficult to acquire users' log-in time. This paper determines users' log-in time law by analyzing users' publishing blog time law each day, which is time law of users reading blog. If two users' time law to publish blog is similar, that is their log-in micro-blog time is similar each day, it indicates that friends' publishing blog 
Table 1. Topological parameters comparison of several social networks.

\begin{tabular}{|c|c|c|c|}
\hline Platform & Average minimum path & Diameter & Clustering parameter \\
\hline \hline Sina micro-blog & 4.32 & 13 & 0.229 \\
\hline Tecent micro-blog & 3.47 & 9 & 0.21 \\
\hline Twitter & - & 6 & 0.106 \\
\hline YouTube & 4.9 & 20 & 0.135 \\
\hline Facebook & 5.69 & 22 & 0.320 \\
\hline
\end{tabular}

time approximates to users' on-line time. The possibility of users' to read his friends' blogs is very large.

For any user $\mu$, we compute the packet sending probability each hour in 24 hours per day. As the rules of sending time, the time rules for user are: assuming the user issues $N$ blogs totally in a day and $i$ denotes each little intervals in 24 hours $(i \in[0, \ldots, 23])$. If $N_{i}$ pieces of blogs are issued in each interval $i$, the packet sending probability is $p_{i}=\frac{N_{i}}{N}$. That is to say, the more pieces of blogs issued by user in the $i_{t h}$ are, the probability of these blogs issued and read in this period are larger. Two -tuples $\langle t, p\rangle$ denotes that the probability of issuing blogs is $p$ in hour $t$, then the packet sending time rule of user is represented by set $p_{t r}$ :

$$
p_{t r}=\left\{<t_{0}, p_{0}>,<t_{1}, p_{1}>, \ldots,<t_{23}, p_{23}>\right\}
$$

\section{Analysis of Transmission and Comment Behavior}

Fig. (3) shows micro-blog distribution of transmission number and comment number. From this figure, micro-blog transmission has large difference because updating information quantity of sina micro-blog is extremely large. Most micro-blog is sunk in information ocean in fragment so the chance to be transmitted is very less. Only small amount of micro-blogs can have more opportunities to transmit. Similar to transmission number, comment number also has significant difference. There is no people or few people to comment most micro-blog. In this sample, $54 \%$ of received comment number of micro-blog is smaller than 10 .

In actual micro-blogs, some users add some famous blog owners but they do not concern these blog owners' information to ignore transmission or evaluation. Thus, it also needs to consider users to treat difference of transmitting behaviors between different friends to illustrate different friends' importance on users. While defining transmitting preference degree between users, it needs analyze users' practical blog transmission from different friending in statistics. That is, it is measured by users' historical transmission rate of his friends' blog. If user $u$ concerns user $v$, to define transmitting preference degree of user $u$ on user $v$ is:

$$
\mathrm{P}_{\text {Retweet }}(u, v)=\frac{R(u, v)}{R(u)}
$$

\section{Algorithm Implementation}

In order to predict total transmission of one micro-blog, and to calculate influencing factors between micro-blog transmission rate and users node, the micro-blog information propagation model is proposed. The core of this model is: to calculate one users' transmission rate of one micro-blog, if a user transmit and accumulate his influencing factors, the total transmission number of one original micro-blog is obtained. The steps in detail:

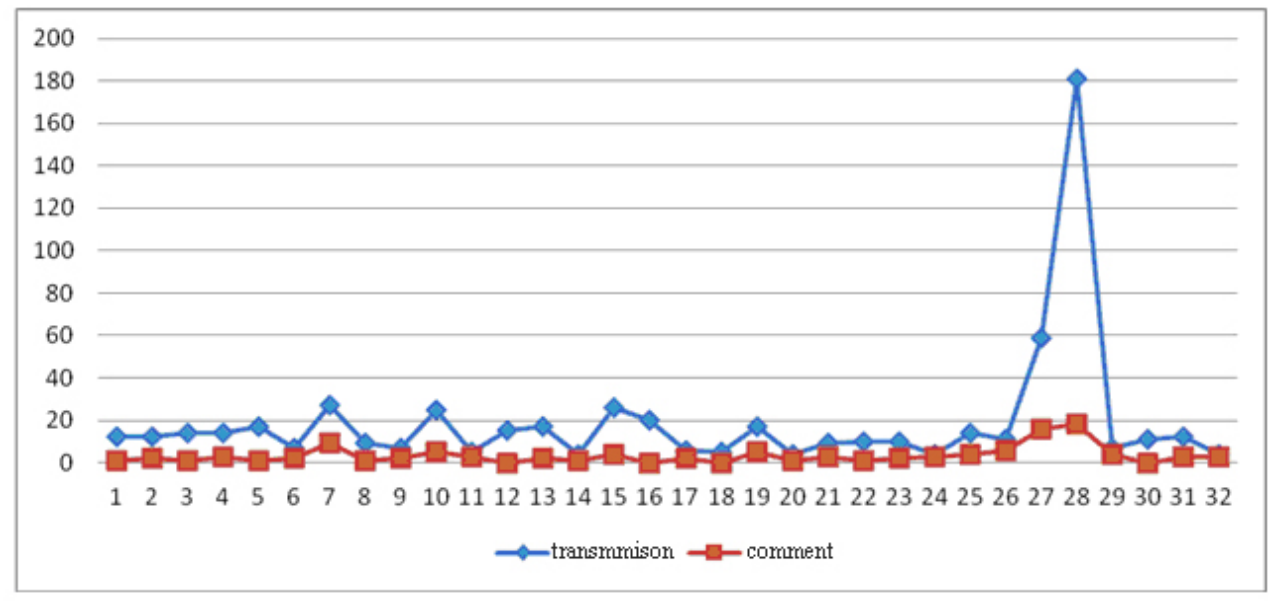

Fig. (3). Distribution of microblog transmission and comment. 
Step 1: One piece micro-blog is broadcast in the network, set the number of transmission as $N=0$, threshold $\lambda=0.5$;

Step 2: The fans node of source node receives the message first and computes transmission rate of fans node $v_{i}$;

Step 3: If the transmission rate of $v_{i}$ is bigger than threshold $\lambda$ this micro-blog will be transmitted and $N=N+A F$ ( $A F$ is the affecting factor of $v_{i}$ ); if it is smaller than this threshold the micro-blog will not be transmitted;

Step 4: Let $i=i+1$, repeat executing step 2 and 3 until all of fans' transmission rate of source node are computed.

To acquire nodes fans list of one awaiting issued microblog, the relationship attribute between node and its fans, the issued micro-blog attribute between node and its fans within one month, the solution process is:

Input: node $v^{\prime}$ and set $V$ composed by its fans nodes, along with one piece of micro-blog $M^{\prime}$ of $v^{\prime}$. Extracting ATT from relation network and obtaining pic, link, len of $M^{\prime} . P_{v_{i}}$ denotes the transmission rate of $v^{\prime}$

Output : Total number of transmission times count

Generate attribute set,

Set count $=0, \lambda=0.5$;

Import to SPSS and analyze regression coefficient;

Generate regression model;

For every follower $v_{i}$

If $v_{i}<\lambda$

Count $+=\operatorname{affect}\left(v_{i}\right)$;

End if

end

ATT includes the transmission results of each fans node issuing micro-blogs for $v^{\prime}$ in a period. The data extraction adopts Java language: the data is stored by structure of $<$ String, Map $<$ String, double []$>$. Then its format to be export to Excel files by SPSS and relative parameters are set for two classification of logic regression analysis. So the regression coefficients are acquired to generate detailed regression formulas.

The attribute of $M^{\prime}$ and each fans node are substituted into regression formula to obtain the transmission rate $P_{v_{i}}$. If $P_{v_{i}}>\lambda$, it denotes $v_{i}$ transmits $M^{\prime}$. The value of current count is added to affecting factor $A F\left(v_{i}\right)$ to obtain current transmission numbers of $M^{\prime}$. When the cycling is over, acquired stable transmission number of $M^{\prime}$ is expected value.

\section{MODEL TESTS AND RESULT ANALYSIS}

This experiment implemented in OS Windows 7, it selects MyEclipse as development tool and compiles Java program to grasp experiment data. We also implement visualized analysis software Matlab to display relation graph of two attributes and software Gephi to analyze complex network to display node relationship graph. Data mining tool SPSS is applied to analyze mathematical model. In order to clearly analyze users' various behavior characteristics influence on information communication in network, we select some relatively active users as experimental objectives according to users' registered time and recently published blog quantity. Micro-blog network data and network topology characteristics in simulation experiment are shown as Table 2.

This test performs information communication and simulation towards different reading threshold probability. The same node will be selected in simulation experiment as initial node of information communication. This node influence is 0.041 and fans number is 607 . Under condition of different reading threshold values $0.75,0.8$ and 0.85 , communication process simulation of information is performed. The following figure is the quantity of reading users and transmitting users change with time under condition that the same user is taken as initial communicator. Abscissa refers to time and ordinate refers to user quantity of reading information and transmitting information in current network.

In Fig. (4), the larger the reading threshold, the smaller the possibility user reads information. From this figure, when reading threshold is smaller, information communication scope is very large. It indicates that there are many users who have read information and there will be more users to transmit information. When $\mathrm{t}=0.85$, information communication will appear stagnation. It means if one user's blog has small probability to be read, its possibility to be transmitted is also small. When $\mathrm{a}=0.75$ and 0.8 , the probability of user to read his friend's blog is large. Therefore, information will be diffused in fast transmitting rate in network at its initial time. Meanwhile, it will pass longer communication time to reach its stable status.

Then, illustrated by 33 micro-blogs of one person, his fans transmission is shown as (Fig. 5). Lateral axis refers to fans' transmitting micro-blog quantity and longitudinal axis refers to the fans quantity to transmit this micro-blog. Based on (Fig. 5), people who seldom transmit this micro-blog are larger and people who tend to transmit friends' micro-blogs are fewer.

Partial intermediate data in fans node transmission microblog contains non-original rate of nodes, average transmission micro-blog number, transmitting percentage, node micro-blog attribute and transmission results, as shown in Table 3.

Above 7 attributes are ranked according to gain rate calculation formula, which is shown as Table 4. It is found that transmitting percentage largely influences transmission rate and text length of micro-blog has less influence on transmitting rate. 

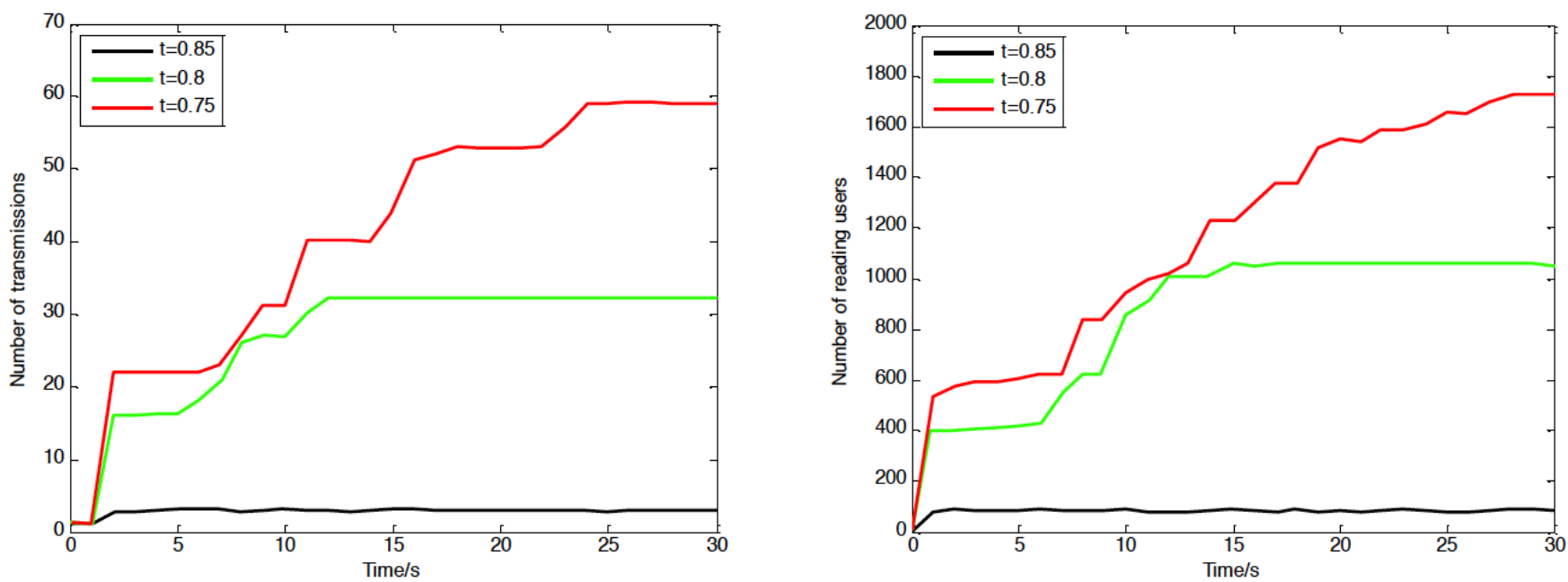

Fig. (4). The effects of user reading habits to message broadcasting.

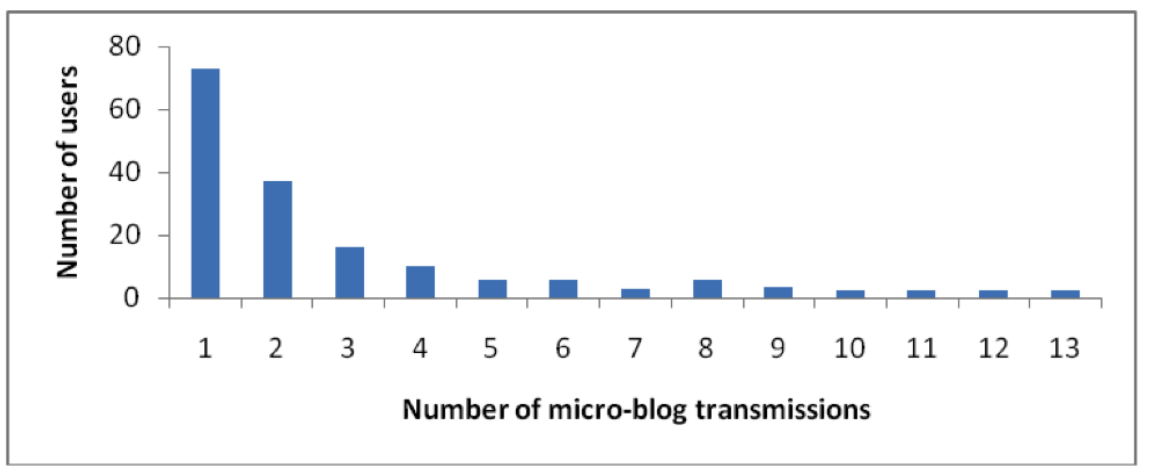

Fig.(5). The relationship between the number of users and micro-blog transmissions.

Table 2. Micro-blog data in messages broadcasting simulation.

\begin{tabular}{|c|c|c|c|c|}
\hline Number of user & Nubmer of relation & Average minimum path & diameter & Clustering papameter \\
\hline \hline 9943 & 210453 & 3.22 & 17 & 0.329 \\
\hline
\end{tabular}

Table 3. Parts of intermediate data.

\begin{tabular}{|c|c|c|c|c|c|c|}
\hline NO. & Non-orginal rate & Average transmission rate & Transmission proprotion & Orginal link & Images & Total length \\
\hline 1 & .864355089 & 13.13333333 & .80564413 & 1 & 1 & 247 \\
\hline 2 & .782363663 & 44.80000000 & .70995664 & 0 & 1 & 242 \\
\hline 3 & .979552210 & 6.200000000 & .52449709 & 0 & 1 & 246 \\
\hline 4 & .942678821 & 12.86666677 & .35498870 & 1 & 1 & 193 \\
\hline 5 & .997416022 & 12.86666600 & .29032258 & 0 & 1 & 183 \\
\hline 6 & .988890099 & 11.86666660 & .23999705 & 0 & 0 & 146 \\
\hline 7 & .991111112 & 7.443333333 & .29023358 & 0 & 1 & 242 \\
\hline 8 & .979122339 & 40.66666660 & .12392257 & 0 & 0 & 143 \\
\hline 9 & .991071458 & 14.87777777 & .19354787 & 1 & 0 & 125 \\
\hline 10 & .998787219 & 27.46666670 & .13642286 & 1 & 1 & 276 \\
\hline
\end{tabular}


Table 4. Ranks of different attributes.

\begin{tabular}{|c|c|c|}
\hline Rank & Name of attribute & Gain rate \\
\hline \hline 1 & Transmission proprotion & 0.334 \\
\hline 2 & Number of images & 0.227 \\
\hline 3 & Number of average transmission & 0.126 \\
\hline 4 & Transmission level & 0.157 \\
\hline 5 & Non-orginal rate & 0.148 \\
\hline 6 & Number of original links & 0.116 \\
\hline 7 & Total length & 0.089 \\
\hline
\end{tabular}

Table 5. Confusion matrix of transmission results.

\begin{tabular}{|c|c|c|c|c|}
\hline Actual Prediction & Transimission & Without transimission & Sum & Recognition rate \\
\hline \hline Transimission & 52 & 37 & 99 & $56.9 \%$ \\
\hline Without transimission & 51 & 866 & 917 & $94.2 \%$ \\
\hline Sum & 103 & 891 & 1000 & $91.3 \%$ \\
\hline
\end{tabular}

To calculate fans transmission rate, according to model 2 , the solution of $P$ is smaller than 0.5 . It means there are 890 non-transmissions and 103 transmissions. From these 1000 fans, there are 188 practical transmissions and confusion matrix of transmitting result is shown as Table 4.

In Table 5, 52 refers to correct transmission prediction number, 35 refers to wrong transmission prediction number, 51 refers to wrong non-transmission prediction number, 862 refers to correct non-transmission prediction number, $56.9 \%$ refers to transmitting recognition rate, $94.2 \%$ refers to nontransmission recognition rate and $91.3 \%$ refers to total recognition rate. It is found that there is no difference on overall recognition rate during simulation. Although transmitting recognition rate is only $56.9 \%$, overall recognition rate is $91.3 \%$. Then, to accumulate influencing factors of transmitting micro-blog fans, transmitting number count 118 is obtained. By substitution of formula, overall transmission number 1522 is obtained. Practical transmission number of this micro-blog is 1805 and error rate is $E \approx 0.14<0.2$. Prediction value basically reflects genuine transmission number.

\section{CONCLUSION}

Ideological and political education confronts challenges and wins opportunities in micro-blog period. Ideological and political educators should actively explore and implement working mode to apply micro-blog to develop ideological and political education. They should take it as beneficial supplement for ideological and political education and actively explore long-term mechanism of ideological and political education of on-line and off-line combination to promote ideological and political education to advance with times and be full of vitality.

This paper is based on SINA micro-blog platform to obtain relationship between users' relationship and microblog information by calling application program access of opening platform and grasping micro-blog web pages. Then, the content information of user and micro-blogs are performed characteristics extraction and analysis. Formalization defines basic definitions of user transmission rate, key nodes influence and information transmission ability, and describes its quantity measurement to further propose a method to construct micro-blog information communication relationship net. In order to test scientificalness and effectiveness of model, under Windows7, this paper adopts Java language to implement our algorithm in program, to calculate text user's influence, reading user's reading probability and transmission preference of transmitting users. To predict communication scope of different initial users' information, overall prediction accuracy rate reaches $88.10 \%$. Since error rate is in hypothesis scope, the model feasibility is proved.

\section{CONFLICT OF INTEREST}

The author confirms that this article content has no conflict of interest.

\section{ACKNOWLEDGMENTS}

This work is supported by the 2013 scientific research project of Beifang University of Nationalities (2013XYZ021), institute of information and system computation science of Beifang University (13xyb01). 


\section{REFERENCES}

[1] X. Chen, T. Lu, L. Guo, and X. Li, "Analysis and empirical study on influence factors of enterprise microblog marketing effect," Journal of Chemical \& Pharmaceutical Research, vol. 6, pp. 1529$1535,2014$.

[2] J. Y. Choeh, H. J. Lee, and J. Eugene, "Exploring time aware features in microblog to measure TV ratings," IEICE Transactions on Information and Systems, vol. 97, pp. 2810-2813, 2014.

[3] T. Hu, Y. Che, and P. Wang, "Empirical research on the effects of IWOM to consumer purchasing intention: based on the microblogging marketing," Advances in Information Sciences \& Service Sciences, vol. 4, pp. 312-319, 2012.

[4] J. Liu, K. Niu, Z. He, and J. Lin, "Analysis of rumor spreading in communities based on modified SIR model in microblog," In: Artificial Intelligence: Methodology, Systems, and Applications, Springer, Berlin, 2014, pp. 69-79.
[5] Z. Qin, M. Xin, and Z. Niu, "A content tendency judgment algorithm for micro-blog platform," In: IEEE International Conference on Intelligent Computing and Intelligent Systems (ICIS), 2010, pp. 168-172.

[6] W. Shoujin, Q. Ying, and L. W. G. Kun, "A study of spreading mode and features of microblogging based on topics," Journal of Intelligence, vol. 6, p. 33, 2013

[7] C. Wu, Y. Du, and C. Su, "Topic recommendation method with finite driving user nodes in micro-blogging," Computer Engineering and Applications, vol. 15, p. 33, 2013.

[8] Y. Li-Xiang, "Communication modes and effects of twitter," Journal of Anhui Normal University, vol. 6, p. 10, 2011.

[9] H. Yu, H. Li, C. Liu, and J. Ge, "The modeling and analysis of microblog forwarding intentions based on a structural equation", In: WIT Transactions on Information and Communication Technologies, vol. 55, pp. 603-611, 2014.

(C) Tang Yeren; Licensee Bentham Open.

This is an open access article licensed under the terms of the (https://creativecommons.org/licenses/by/4.0/legalcode), which permits unrestricted, noncommercial use, distribution and reproduction in any medium, provided the work is properly cited. 